\title{
Technical note: Use of accelerometers to describe gait patterns in dairy calves
}

\author{
A. M. de Passillé, ${ }^{* 1} M$ M. B. Jensen, $†$ N. Chapinal, $\ddagger$ and J. Rushen* \\ ${ }^{*}$ Pacific-Agri-Food Research Centre, Agriculture and Agri-Food Canada, PO 10006947 Highway 7, Agassiz, BC, Canada, V0M 1A0 \\ †University of Aarhus, Faculty of Agricultural Sciences, Department of Animal Health and Bioscience, Blichers Allé 20, PO Box 50, DK-8830 Tjele, \\ Denmark \\ $\ddagger$ Animal Welfare Program, Faculty of Land and Food Systems, University of British Columbia, Vancouver, BC, Canada
}

\begin{abstract}
Developments in accelerometer technology offer new opportunities for automatic monitoring of animal behavior. Until now, commercially available accelerometers have been used to measure walking in adult cows but have failed to identify walking in calves. We described the pattern of acceleration associated with various gaits in calves and tested whether measures of acceleration could be used to count steps and distinguish among gait types. A triaxial accelerometer (sampling at 33 readings $/ \mathrm{s}$ with maximum measurement at $\pm 3.2 \mathrm{~g}$ ) was attached to 1 hind leg of 7 dairy calves, and each calf was walked to a familiar large arena $(29.1 \times 4.8 \mathrm{~m})$ and encouraged to walk and run for 8 to 10 min while being video recorded. The video recordings were watched in slow motion and a total of 54 recordings of 3 to $6 \mathrm{~s}$ duration of either galloping $(\mathrm{n}=21)$, trotting $(\mathrm{n}=13)$, or walking $(\mathrm{n}=21)$ were identified and the number of steps were counted. Accelerometer data was then analyzed for each gait. Steps could be clearly identified by changes in the acceleration in the forward and vertical axes and vector sum, but less clearly in the lateral axis. The number of steps counted using the forward axis was highly correlated with the number observed from the video recordings. Galloping, trotting, and walking differed significantly in the median interpeak intervals in acceleration in the forward axis and in the vector sum of the acceleration in the 2 axes. Interpeak intervals could be used to discriminate among the 3 gaits, although walking was most clearly distinguished from galloping. Automated measures of acceleration of the leg in the forward and vertical dimensions can be used to count steps and classify gaits of calves.
\end{abstract}

Key words: welfare, accelerometer, locomotion, dairy calf

Received September 21, 2009.

Accepted March 16, 2010.

${ }^{1}$ Corresponding author: Annemarie.Depassille@agr.gc.ca
Developments in sensor technology offer new opportunities for automatic monitoring of behavior in animals. Dairy calves perform a range of locomotor behaviors, including walking, trotting, and galloping (Jensen et al., 1998). Measures of activity may be useful to assess welfare and health (Rushen et al., 2008); therefore, it would be an advantage to be able to measure locomotor behavior automatically. Watanabe et al. (2005) showed that accelerometers could distinguish between walking, trotting, and running in cats. Commercially available accelerometers attached to the legs can distinguish walking patterns in adult cows (Munksgaard et al., 2006) but failed to identify walking in calves (White et al., 2008; Trénel et al., 2009). However, the commercial availability and practical use of accelerometers appears to have gone ahead of our basic knowledge of the patterns of acceleration associated with movement patterns, and this may explain the reported failures of accelerometers to detect movement patterns in calves.

The aims of the present study are to test whether measures of acceleration of the legs of calves could be used to measure the number of steps and to determine the ability to distinguish among different types of gait (walking, trotting, and galloping).

Seven female Holstein calves (age range: 70-92 d; weight range: $78-116 \mathrm{~kg}$ ) were fitted with a triaxial accelerometer (Hobo Pendant G Acceleration Data Logger, Onset Computer Corporation, Pocasset, MA), described in Moreau et al. (2009), attached with Vet Wrap (Co-Flex, Andover Coated Products Inc., Salisbury, MA) to the lateral side of the left hind leg. The accelerometer could measure a maximum of $3.2 \mathrm{~g}$ with an accuracy of $\pm 0.075 \mathrm{~g}$ at $25^{\circ} \mathrm{C}$ and an accuracy of $\pm 0.105 \mathrm{~g}$ from $-20^{\circ} \mathrm{C}$ to $70^{\circ} \mathrm{C}$. The accelerometer was set to measure the acceleration at 33 readings/s. This was based on calculations that showed that this was the maximum sampling rate that we could use that would allow the memory of the accelerometer to store data for the expected duration of the trials.

Immediately after being fitted with the accelerometer, the calves were individually guided along a corridor that 

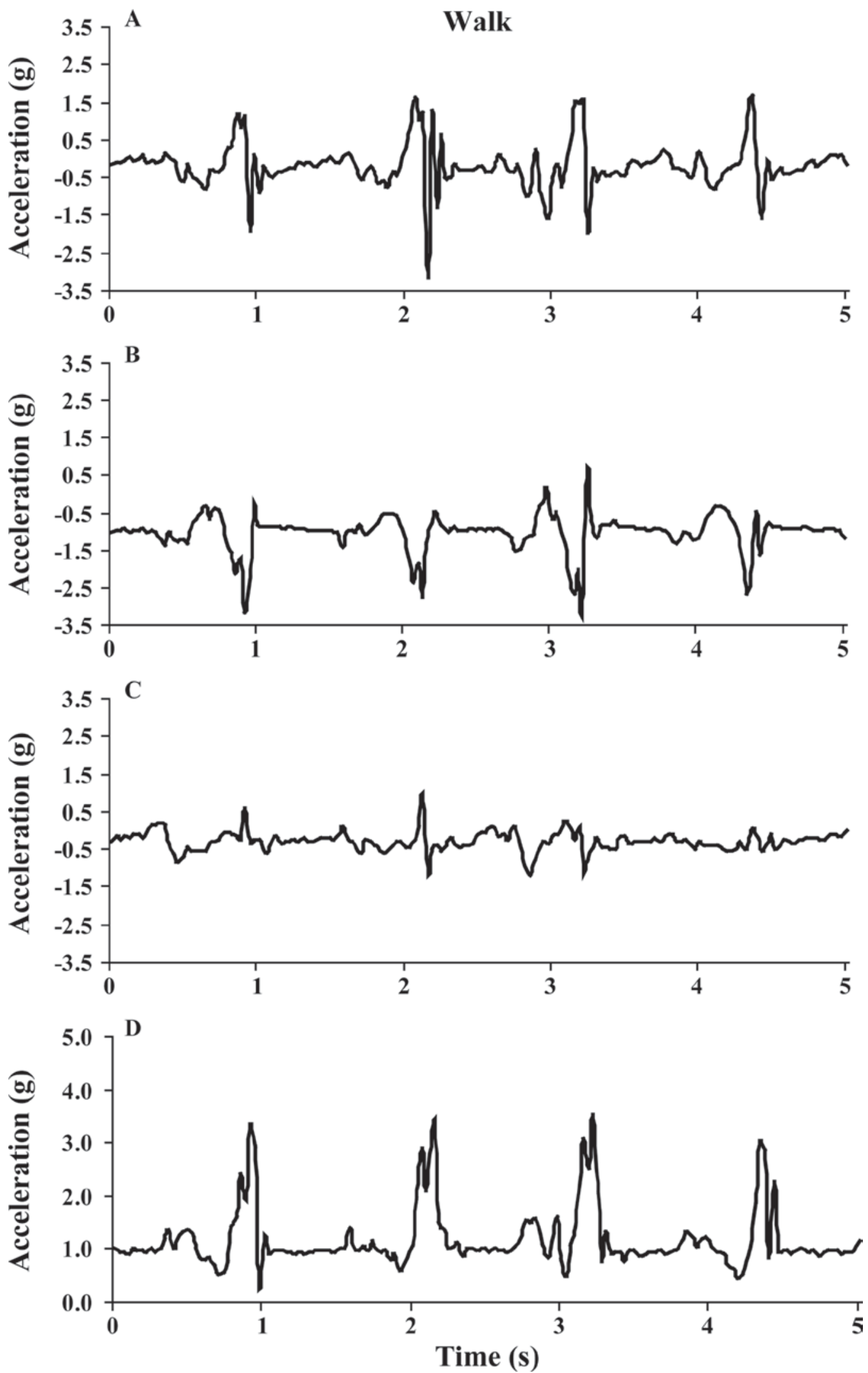

Figure 1. An example of acceleration in the A) forward, B) vertical, and C) lateral axes and D) the vector sum of the forward and vertical axes during 1 period when a calf was walking, trotting, and galloping. 
Trot
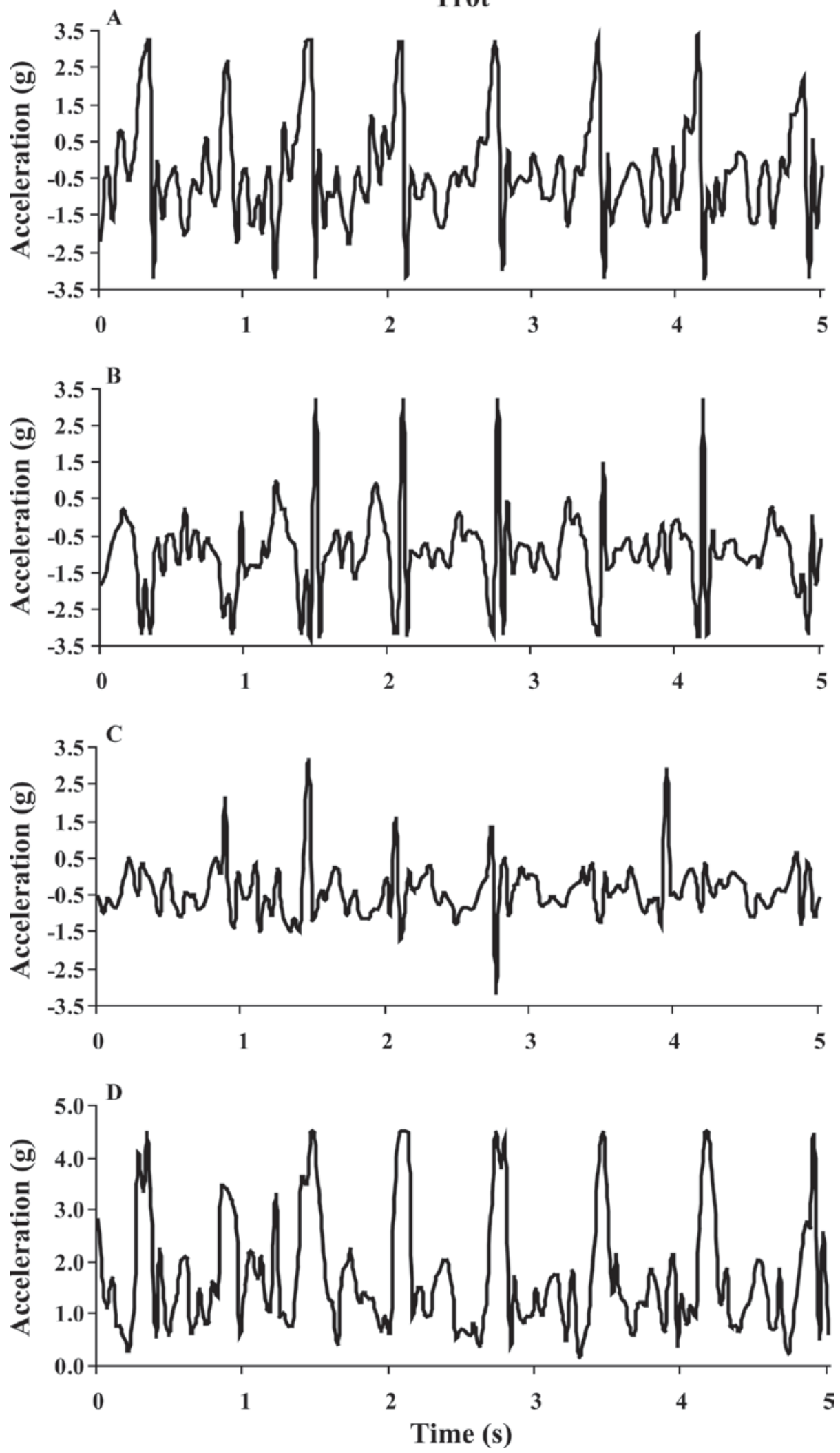

Figure 1(Continued). An example of acceleration in the A) forward, B) vertical, and C) lateral axes and D) the vector sum of the forward and vertical axes during 1 period when a calf was walking, trotting, and galloping. 
DE PASSILLÉ ET AL.
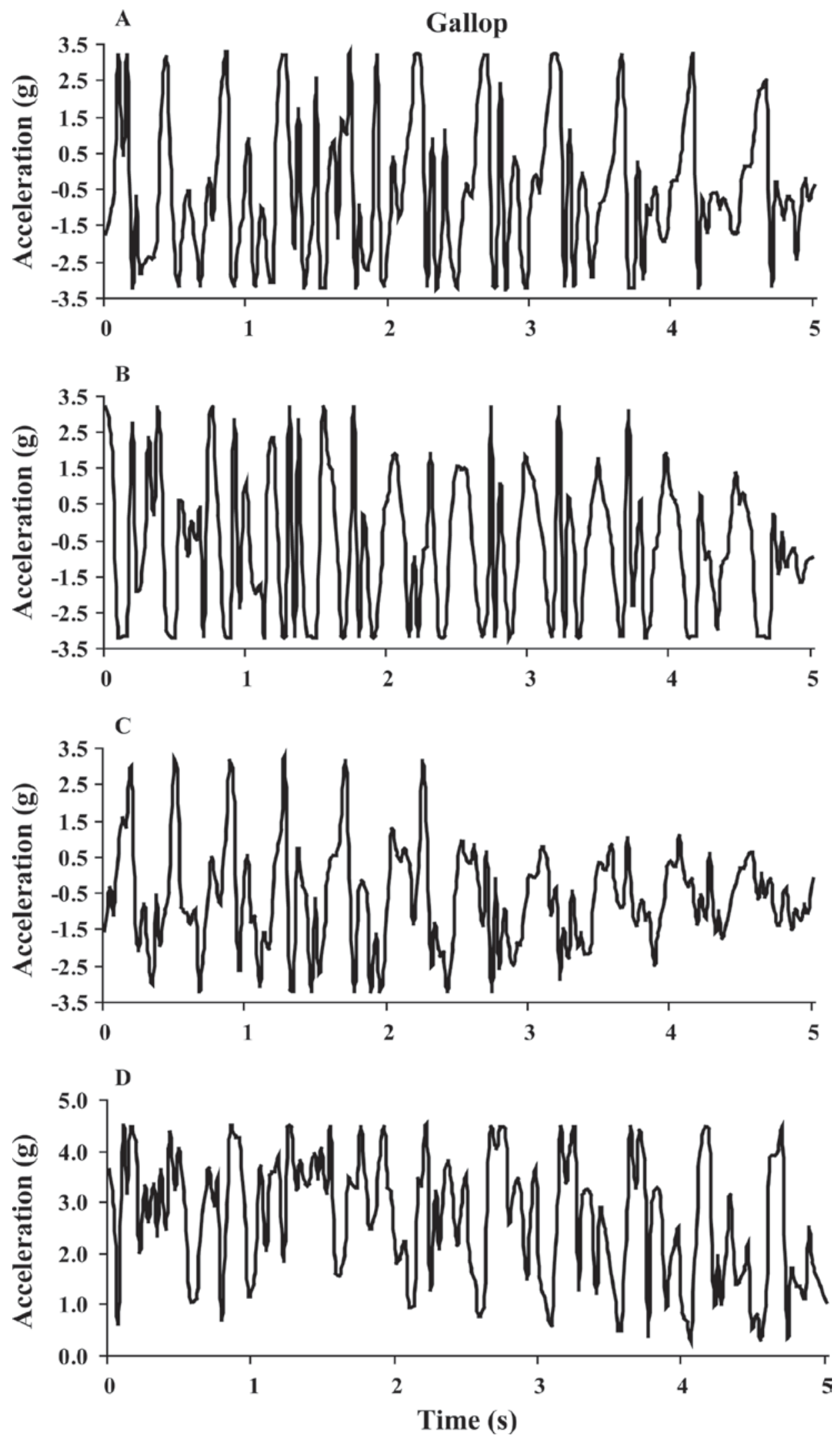

Figure 1 (Continued). An example of acceleration in the A) forward, B) vertical, and C) lateral axes and D) the vector sum of the forward and vertical axes during 1 period when a calf was walking, trotting, and galloping. 
led to an arena $(29.13 \times 4.77 \mathrm{~m})$ with concrete ridged floors and high walls that prevented visual contact with other calves to minimize disturbances. Before the trial, the calves had been accustomed on 7 previous occasions to being walked to this arena and released there for 20 min. Depending on the position of the calf's pen, the distance walked along the corridor varied from 15 to 25 $\mathrm{m}$. Once in the arena, calves were encouraged to run with a person running by their side. After 8 to $10 \mathrm{~min}$, the calves were taken back to their pens. A hand-held digital camcorder (Sony DCRSR100 HDD Handycam Camcorder, Sony Corp., Park Ridge, NJ) was used to record the locomotion of the calves at 30 frames/s when they were in the corridor and the arena, keeping the leg with the accelerometer in view. The video recorder and the accelerometers were initially synchronized by setting the clock of the video to match the time on the computer that was used to launch the accelerometers.

The video recordings were observed independently by 2 observers using continuous observation. Based on the behavioral definition of each gait (Jensen, 1999), the observers identified sequences of continuous locomotion lasting between 3 and $6 \mathrm{~s}$ and categorized these into walking (21 sequences from 7 calves), trotting (13 sequences from 5 calves), or galloping (21 sequences from 7 calves) with $100 \%$ agreement. The numbers of steps within each sequence were counted twice by watching the videos frame by frame. Graphs of the acceleration data from the corresponding time periods were visually inspected and the numbers of steps were counted using the peaks in acceleration in the forward axis (Figure 1). Two observers were used; one observer had participated in the experiment, whereas the second observer was new. The average interstep interval for each sequence was calculated by finding the time of the first step and the time of the last step in the forward axis and dividing this by the number of interstep intervals.

The vector sum of the acceleration in the forward and vertical axes and the vector sum of acceleration of the forward, lateral, and vertical axes were calculated for each sequence as the square root of the sum of the squares of the acceleration for each axis. Because steps were most clearly seen in acceleration in the vertical and forward axes and less so in the lateral axis (see below), we based analyses of the number of steps on the acceleration in the forward axis, the vertical axis, and the vector sum of these 2 axes. The vector sum of the acceleration in the 3 axes was averaged over time for each sequence and used as an estimation of energy expenditure (Green et al., 2009).

Spearman correlations were calculated between the number of peaks counted in the acceleration data by the 2 observers and between the average number of peaks in the acceleration data and the number of steps observed from the videos. The interpeak intervals and the vector sum of acceleration were not normally distributed and this could not be corrected by transformations. Therefore, a nonparametric test was used for the analysis. Values for both variables were first ranked within calf (to reduce differences between calves). The overall differences between the gaits were first tested with a Kruskal-Wallis test. Where there was an overall significant difference, pairwise comparisons between the gaits was then done using a Wilcoxon rank-sum test.

Figure 1 shows a representative example of each of the 3 types of gait, with the acceleration in the forward, vertical, and lateral axes and the vector sum of the forward and vertical axes. During walking, individual steps were clearly apparent in both the forward and vertical axes. The positive and negative peaks in both the forward and vertical axes were associated with the hoof descending and pushing off from the ground. These peaks were interspersed by periods of no or very little acceleration, most likely associated with the swing phase (Tanida et al., 2008). Changes in lateral acceleration were also associated with steps but these were much less obvious. During trotting a similar pattern could be seen except that the magnitude of the peaks and the magnitude of the acceleration between peaks were larger. During galloping, frequent and distinct peaks of acceleration were observed in all 3 axes. During both galloping and trotting, the recorded magnitude of the acceleration during these peaks often reached the maximum that could be recorded from the accelerometer $(3.2 \mathrm{~g})$.

The number of peaks in acceleration was highly correlated between the 2 observers for the forward axis (r $=0.95 ; \mathrm{n}=54 ; P<0.001)$, the vertical axis $(\mathrm{r}=0.87$; $\mathrm{n}=54 ; P<0.001$ ), and the vector sum of forward and vertical axes $(\mathrm{r}=0.94 ; \mathrm{n}=54 ; P<0.001)$. The observed number of steps from the video was most highly correlated with the number of peaks in the acceleration (averaged across the 2 observers) in the forward axis ( $\mathrm{r}$ $=0.92 ; \mathrm{n}=54 ; P<0.001)$, the vertical axis $(\mathrm{r}=0.93$; $\mathrm{n}=54 ; P<0.001$ ), and the vector sum of the forward and vertical axes $(\mathrm{r}=0.88 ; \mathrm{n}=54 ; P<0.001)$.

The distribution of interpeak intervals in the forward axis differed among the 3 gait types (Figure 2; KruskalWallis test: $P<0.001)$. There was no overlap between walking and galloping, and these 2 differed significantly (Wilcoxon rank-sum test: $P<0.001$ ). However, the distribution of interpeak intervals when the calves were trotting overlapped with both walking and galloping. Nevertheless, trotting differed from galloping (Wilcoxon rank-sum test: $P<0.001$ ) and walking (Wilcoxon ranksum test: $P<0.001)$. A cut-off value of $0.53 \mathrm{~s}$ correctly classified all instances of galloping but misclassified 1 (8\%) sequence of trotting, and a cut-off value of $0.76 \mathrm{~s}$ 
misclassified 2 sequences (15\%) of trotting and $2(9.5 \%)$ sequences of walking.

The vector sum of acceleration in the 3 axes (expressed as average per sequence) also differed among the 3 gaits (Figure 2; Kruskal-Wallis test: $P<0.001$ ).

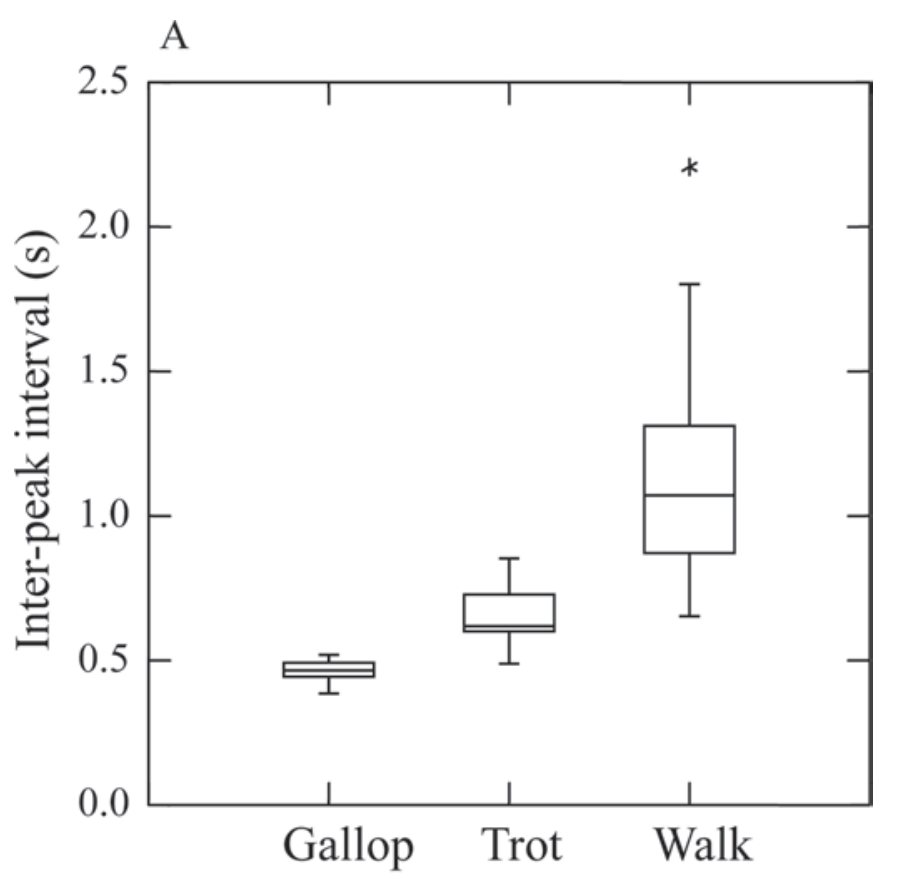

B

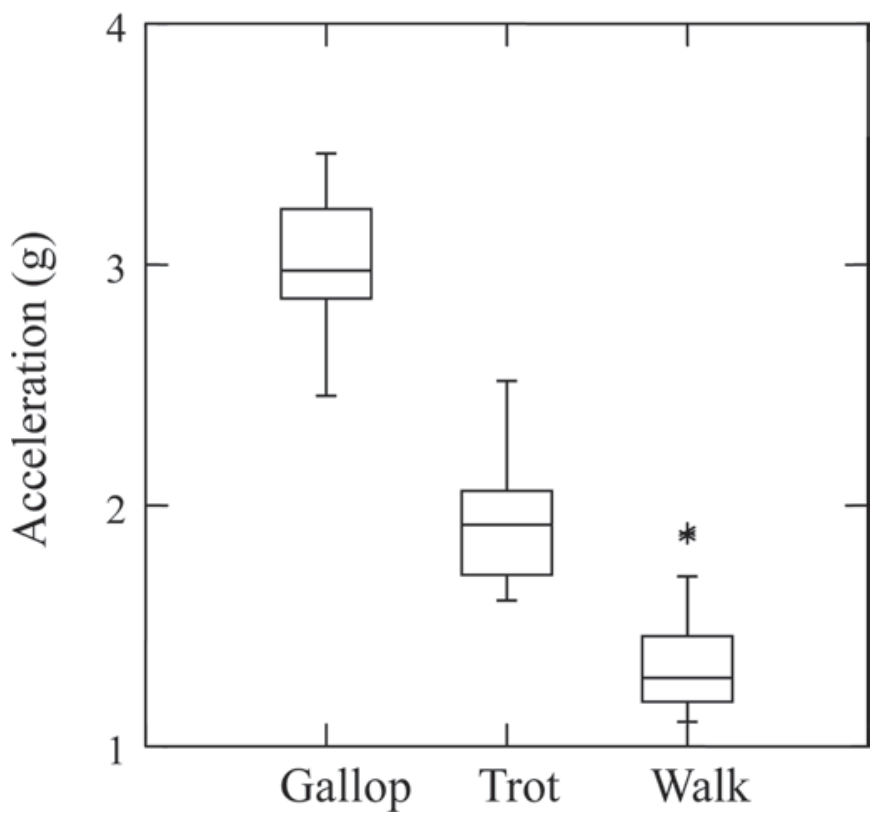

Figure 2. Box plots showing the median, 25th and 75th percentiles, and minimum and maximum values and any outliers for A) the interpeak interval in acceleration in the forward axis and B) the vector sum of the acceleration in the 3 axes (expressed as a mean per sequence) for sequences of galloping, trotting, and walking. Asterisk indicates a likely outlier.
There was no overlap between walking and galloping, and these 2 differed significantly (Wilcoxon rank-sum test: $P<0.001)$. However, the distribution of measures when the calves were trotting overlapped with both walking and galloping (Figure 2). Yet, trotting differed significantly from both galloping (Wilcoxon rank-sum test: $P<0.001$ ) and walking (Wilcoxon rank-sum test: $P<0.001)$. The vector sum of the 3 axes was highly correlated with the interpeak interval $(\mathrm{r}=-0.98 ; \mathrm{n}=$ 54; $P<0.001)$.

The results suggest that data from accelerometers attached to the leg of a calf could be used to count the number of steps taken and to classify the locomotion into walking, trotting, or galloping with a high degree of accuracy. The interstep interval was shortest for galloping, longest for walking, and intermediate for trotting. This variable alone was quite accurate in classifying the selected sequences of locomotion into galloping, walking, or trotting. Trotting and walking were found to be the most likely to be misclassified with each other. Galloping was the most easily distinguished. The differences between sequences in mean interpeak intervals were greatest for walking and relatively small for galloping. This indicates that the speed of walking varies more than does the speed of galloping. Research on other species has shown that the vector sum of the acceleration in 3 dimensions can provide a reasonably accurate estimate of energy expenditure when animals are moving (Green et al., 2009). Our results show that the vector sum of the acceleration was highest in galloping, intermediate in trotting, and lowest in walking, which relates to the likely degree of energy expenditure in these 3 gaits. The vector sum of the acceleration was correlated highly with the number of steps, suggesting that this measure may be used to estimate overall activity in tests such as the open field (Rushen et al., 2008).

However, our ability to estimate energy expenditure and to differentiate trotting from galloping and walking was affected by the limit on the maximum acceleration that could be measured by the accelerometers that we used. The limit of $3.2 \mathrm{~g}$ was reached often during galloping and trotting but rarely reached for walking. Thus, estimates of energy expenditure are most accurate when the animal is walking but could underestimate energy expenditure when trotting and galloping. It is likely that we would have obtained a much better differentiation of the 3 gaits had we been able to include the actual magnitude of the acceleration involved.

The accelerometers do not measure acceleration continuously but take point measures of acceleration at a predetermined rate (the sampling rate). The ability of accelerometers to identify behavior patterns increases with an increase in rate that the accelerometers sample 
the actual acceleration (Moreau et al., 2009). The sampling rate is particularly likely to affect the accuracy of differentiating gaits and counting steps. Peak accelerations, especially during galloping, were very brief and often shown only during a single time sample. This shows that our sampling rate of 33 readings/s was close to the minimum necessary to detect steps. This may explain the failure of some accelerometers that use lower sampling rates to identify behavior patterns; for example, Trénel et al. (2009) could not identify walking in calves using a sampling rate of 8 readings/s. However, these accelerometers store the data in memory until the data are downloaded. With the limited memory available on such portable devices, there is inevitably a trade-off between the sampling rate and the length of time that the devices can be used (Wilson et al., 2008). One way to increase the sampling rate would be to reduce the load on the memory of the accelerometer by reducing the number of axes measured. The steps could be clearly seen in the measures of acceleration in the forward axis alone. Measuring the acceleration in the vertical axis did not improve the correlation with actual steps, and steps could not easily be seen in acceleration in the lateral axis except when the calves were galloping. Thus, the sampling rate could be increased by recording acceleration only in the forward axis. Our study was based on using examples of locomotion that were chosen because they consisted of only 1 type of gait. Our results show that measures of acceleration can differentiate the gait patterns in these circumstances. However, complete validation would require that the method be able to differentiate among gaits when combined in the same period of time.

\section{ACKNOWLEDGMENTS}

We thank Emilie Bullo, Sara Woods, and Gosia Zdanowicz (Agriculture and Agri-Food Canada, Agas- siz, British Columbia, Canada) and Andrea de Paula Vieira (University of British Columbia, Vancouver, British Columbia, Canada) for their help. Funding was from the Natural Sciences and Engineering Research Council of Canada (Ottawa, Ontario, Canada).

\section{REFERENCES}

Green, J. A., L. G. Halsey, R. P. Wilson, and P. B. Frappell. 2009. Estimating energy expenditure of animals using the accelerometry technique: Activity, inactivity and comparison with the heart-rate technique. J. Exp. Biol. 212:471-482.

Jensen, M. B. 1999. Effects of confinement on rebounds of locomotor behaviour of calves and heifers, and the spatial preferences of calves. Appl. Anim. Behav. Sci. 62:43-56.

Jensen, M. B., K. S. Vestergaard, and C. C. Krohn. 1998. Play behaviour in domestic calves kept in pens: The effect of social contact and space allowance. Appl. Anim. Behav. Sci. 56:97-108.

Moreau, M. S., A. Siebert, and E. Schlecht. 2009. Use of a tri-axial accelerometer for automated recording and classification of goats' grazing behaviour. Appl. Anim. Behav. Sci. 119:158-170.

Munksgaard, L., C. G. Reenen, and R. Boyce. 2006. Automatic monitoring of lying, standing and walking behavior in dairy cattle. J. Anim. Sci. 84(Suppl.):304.

Rushen, J., A. M. de Passillé, M. A. G. von Keyserlingk, and D. M. Weary. 2008. The Welfare of Cattle. Springer, Dordrecht, the Netherlands.

Tanida, H., Y. Koba, M. Toze, I. Ishii, J. Rushen, and A. M. de Passille. 2008. The effect of hoof trimming on dairy cow gait, assessed by 3-dimensional acceleration sensing. Page 194 in Proc. 15th Symp. and 7th Int. Conf. on Lameness in Ruminants, June 9-13, 2008, Kuopio, Finland. Savonia Univeristy of Applied Sciences, Kuopio, Finland.

Trénel, P., M. B. Jensen, E. L. Decker, and F. Skjøth. 2009. Technical note: Quantifying and characterizing behavior in dairy calves using the IceTag automatic recording device. J. Dairy Sci. 92:33973401.

Watanabe, S., M. Izawa, A. Kato, Y. Ropert-Coudert, and Y. Naito. 2005. A new technique for monitoring the detailed behaviour of terrestrial animals: A case study with the domestic cat. Appl. Anim. Behav. Sci. 94:117-131.

White, B. J., J. F. Coetzee, D. G. Renter, A. H. Babcock, D. U. Thomson, and D. Andresen. 2008. Evaluation of 2-dimensional accelerometers to monitor behavior of beef calves after castration. Am. J. Vet. Res. 69:1005-1012.

Wilson, R. P., E. L. C. Shepard, and N. Liebsch. 2008. Prying into the intimate details of animal lives: Our use of a daily diary on animals. Endang. Species Res. 4:123-137. 